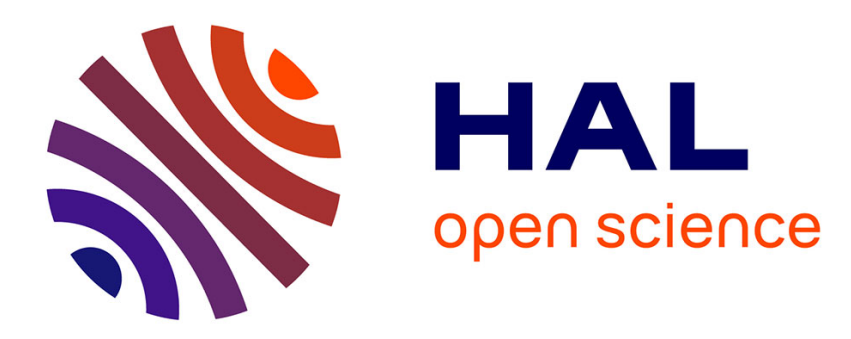

\title{
Pour une pratique humaniste de l'évaluation en ASH
}

\author{
Nicole Mencacci
}

\section{To cite this version:}

Nicole Mencacci. Pour une pratique humaniste de l'évaluation en ASH. La nouvelle revue de l'adaptation et de la scolarisation, 2009, 46, pp.191-202. hal-02436832

\section{HAL Id: hal-02436832 \\ https://hal.science/hal-02436832}

Submitted on 13 Jan 2020

HAL is a multi-disciplinary open access archive for the deposit and dissemination of scientific research documents, whether they are published or not. The documents may come from teaching and research institutions in France or abroad, or from public or private research centers.
L'archive ouverte pluridisciplinaire HAL, est destinée au dépôt et à la diffusion de documents scientifiques de niveau recherche, publiés ou non, émanant des établissements d'enseignement et de recherche français ou étrangers, des laboratoires publics ou privés. 
Nicole Mencacci

Maître de Conférences

Université Nice Sophia Antipolis, Ecole Interne IUFM

UMR P3 ADEF (Apprentissage, Didactique, Evaluation, Formation)

\section{Pour une pratique humaniste de l'évaluation en ASH $^{1}$}

\section{Introduction : l'évaluation, une pratique quotidienne de l'enseignant}

Evaluer est un mot qui revient souvent dans le quotidien de l'enseignant du premier et du second degré, de classe ordinaire ou de classe spécialisée. C'est une compétence qu'il doit mettre en œuvre dans sa pratique professionnelle, à de multiples occasions, et de manière variée. D'ailleurs, depuis 2007, le cahier des charges de la formation des enseignants fait d'«évaluer les élèves », l'une des dix compétences que le futur professeur doit maîtriser de manière suffisante pour pouvoir commencer à enseigner. L'importance de l'évaluation rend alors nécessaire que l'on en clarifie la signification : que veut dire évaluer ?

La mesure est « le plus souvent la seule opération mise en avant dans l'action d'évaluer » (Lecointe, 1977, p. 23). Pourtant, depuis $1967^{2}$ et jusque de nos jours, nombre de chercheurs en évaluation, américains, canadiens et européens ${ }^{3}$ ont contribué depuis à ce que Cardinet (1979) a appelé un élargissement de l'évaluation. En 1997, Bonniol et Vial ont distingué trois conceptions de l'évaluation, « trois entrées différentes dans la pratique évaluative » (Vial, 2001, p. 9), non hiérarchisables parce que centrées sur des objets différents. Ces trois conceptions ont été l'objet de nombreuses recherches. Historiquement, la première conception étudiée est « l'évaluation-mesure » (ibid., p. 44) laquelle se focalise sur les produits de l'enseignement pour déterminer leur degré de conformité à la norme. La deuxième est «l'évaluation-gestion » (ibid.), centrée sur les procédures d'apprentissage des élèves. Evaluer y signifie gérer les situations d'enseignement pour améliorer les apprentissages : l'évaluation formative et formatrice en sont des exemples. La troisième signification est beaucoup moins connue quoique très souvent pratiquée - y compris à notre insu - c'est «l'évaluation- problématisation » (ibid., p. 45), centrée sur les «dynamiques de changement [des élèves] dans la relation [à l'enseignement] » (ibid., p. 10). Ici, évaluer c'est questionner le sens de ce qui est dit et de ce qui est fait.

\footnotetext{
${ }^{1}$ Cet article a fait l'objet d'une contribution lors de la journée d'études organisée, le 3 décembre 2008 à Draguignan, par l'Ecole Interne IUFM de l'Université de Nice Sophia Antipolis.

2 avec Cronbach et Scriven

${ }^{3}$ Avec entre autres, Bloom, Allal, Ardoino, Berger, Bonniol, De Ketele, Nunziati, Amigues, Vial, Scallon, Abrecht, Figari, Jorro, Mottier-Lopez...
} 
Les trois conceptions de l'évaluation vont être successivement présentées. Elles seront accompagnées de questionnements qui permettront au fur et à mesure d'aborder la problématique suivante: comment l'évaluation peut-elle devenir, en ASH, une pratique humaniste qui vise pour l'élève, la restauration de l'image de soi, de l'estime de soi et du désir d'apprendre, et en même temps et de manière indissociable la construction du rapport $\mathrm{au}(\mathrm{x})$ savoir(s)?

\section{L'évaluation comme mesure des acquis}

Les sollicitations institutionnelles pour le contrôle et la mesure des acquis sont historiquement nombreuses. Depuis 1969, avec l'abandon du contrôle sommatif et final, l'enseignant du primaire et du second degré doit pratiquer le contrôle continu de manière à renseigner un bulletin scolaire régulièrement transmis aux parents. En 1989, les Evaluations Nationales sont ajoutées au contrôle continu, à trois niveaux des premier et second degrés, avec une fonction diagnostique. En 1990, dans le premier degré, le bulletin scolaire est remplacé par le livret scolaire et couvre les trois cycles. Les acquis des élèves y sont évalués, par le biais non plus de leurs connaissances, mais de leurs compétences. A partir de la rentrée 2009, un livret supplémentaire, le livret individualisé de compétences, évaluera, en trois paliers au cours de la scolarité obligatoire (CE1 -16 ans), le degré d'acquisition des sept compétences du socle commun de 2006. Pour les élèves à Besoins Educatifs Particuliers (BEP), les compétences ciblées dans les PPRE ${ }^{4}$, dans les Projets d'aide spécialisée et dans les $\mathrm{PPS}^{5}$ sont régulièrement évaluées. Parallèlement à cela, dans le second degré, des examens diplômants sanctionnent les acquis des élèves : le $\mathrm{BEPC}^{6}$ en collège, le $\mathrm{CFG}^{7}$ en $\mathrm{SEGPA}^{8}$, et depuis 1808 , le baccalauréat.

Le tableau ci-dessous récapitule ce que l'institution attend des enseignants en 2009, concernant l'évaluation des acquis des élèves.

\footnotetext{
${ }^{4}$ Programme Personnalisé de Réussite Educative

${ }^{5}$ Projet Personnalisé de Scolarisation

${ }^{6}$ Brevet d'Etudes du Premier Cycle du second degré

${ }^{7}$ Certificat de Formation Générale

${ }^{8}$ Section d'Enseignement Général et Professionnel Adapté
} 


\begin{tabular}{|c|c|}
\hline PREMIER DEGRE & SECOND DEGRE \\
\hline \multicolumn{2}{|c|}{ Contrôle continu } \\
\hline \multicolumn{2}{|c|}{$\begin{array}{c}\text { Conception par l'enseignant d'exercices scolaires réguliers mettant en jeu des compétences du socle commun travaillées en } \\
\text { cours. } \\
\text { Notation par l'enseignant des exercices et/ou évaluation du niveau d'acquisition des connaissances et des compétences } \\
\rightarrow \text { évaluation interne }\end{array}$} \\
\hline \multirow[b]{2}{*}{$\begin{array}{c}\text { Livret scolaire } \\
\text { Fréquence déterminée par le conseil des maîtres } \\
\text { Commun à une circonscription ou à une école } \\
\text { Un livret pour chacun des trois cycles } \\
\text { Renseigné à partir des résultats de l'évaluation continue } \\
\text { Détermine le niveau d'acquisition de chaque compétence du } \\
\text { socle à la fin de chaque période, et à la fin de l'année } \\
\text { Indique les décisions d'orientation du conseil des maîtres. } \\
\rightarrow \text { évaluation interne. }\end{array}$} & Bulletin scolaire \\
\hline & $\begin{array}{c}\text { Trimestriel } \\
\text { Commun à un établissement } \\
\text { Renseigné à partir des résultats de l'évaluation continue } \\
\text { Détermine la moyenne trimestrielle des notes obtenues dans } \\
\text { chaque matière, signale la moyenne de la classe, donne une } \\
\text { moyenne d'ensemble et un avis général des professeurs } \\
\text { (tableau d'honneur, félicitations, encouragements...). } \\
\text { Indique les décisions d'orientation du conseil de classe. } \\
\rightarrow \text { Evaluation interne }\end{array}$ \\
\hline \multicolumn{2}{|c|}{ Evaluations Nationales } \\
\hline \multicolumn{2}{|c|}{$\begin{array}{c}\text { CE1, CM2 et Seconde. } \\
\text { Evaluations bilan et non plus « diagnostic » } \\
\text { Standardisation nationale des items et des conditions de passation } \\
\text { Correction des items par l'équipe pédagogique } \\
\text { Traitement informatique des résultats, transmis aux différentes instances départementales, académiques, nationales, et } \\
\text { rendus partiellement publics. } \\
\rightarrow \text { Evaluation externe et interne }\end{array}$} \\
\hline \multicolumn{2}{|c|}{ Livret individuel de compétences } \\
\hline \multicolumn{2}{|c|}{$\begin{array}{l}\text { Standardisation nationale du livret } \\
\text { Evaluation du niveau d'acquisition de chacune des sept compétences du socle commun à trois paliers de la scolarité } \\
\text { obligatoire (CE1-16 ans) par l'équipe pédagogique } \\
\rightarrow \text { Evaluation interne et externe }\end{array}$} \\
\hline & $\begin{array}{l}\text { Examens délivrant un diplôme } \\
\text { BEPC } \\
\text { CFG } \\
\text { Baccalauréat } \\
\rightarrow \text { Evaluation interne et externe avec dossier de l'élève }\end{array}$ \\
\hline $\begin{array}{r}\text { Programmes ou p } \\
\text { pour les élèves en difficu }\end{array}$ & $\begin{array}{l}\text { jets individualisés } \\
\text { s scolaires ou handicapés }\end{array}$ \\
\hline \multicolumn{2}{|c|}{$\begin{array}{l}\text { PPRE et PPS : Evaluation fines des difficultés d'acquisition d'une ou plusieurs compétences du socle, y compris par le biais } \\
\text { de tests } \\
\qquad \begin{array}{l}\text { Evaluations collectives et/ou pluridisciplinaires internes }\end{array}\end{array}$} \\
\hline
\end{tabular}

Modalités d'évaluation des acquis des élèves en 2009 


\subsection{Le souci de contrôle de l'institution}

Cette recension rapide fait apparaître depuis les années 70, le souci de l'institution d'organiser de plus en plus fréquemment dans la scolarité d'un élève, le contrôle et la mesure de ses acquis. Y sont combinées, des évaluations des connaissances et des compétences, internes et externes, individualisées ou standardisées, en mode «continu » ou en mode « bilan », diplômantes ou non. Elles ont quatre destinataires :

- les élèves, qui ont connaissance du niveau d'acquisition de leurs compétences,

- les enseignants, qui font régulièrement le point sur les acquis des élèves, leurs progrès et difficultés, et peuvent alors engager des remédiations et prendre des décisions d'orientation,

- les parents, qui sont régulièrement informés du travail de leur enfant, de la classe et de l'établissement, en évitant autant que possible une mise en concurrence incontrôlée des lieux d'enseignement,

- l'institution, à ses niveaux départemental, académique et national. Les évaluations des acquis deviennent ici des instruments de pilotage du système éducatif - préparation des plans académiques et départementaux de formation des enseignants. Ce sont aussi des instruments de décisions nationales de politique éducative.

On remarque que le contrôle institutionnel est présent à tous les niveaux du cursus, et bien souvent, plusieurs fois pour un même niveau. Même si les professionnels de l'Education peuvent l'interpréter comme la marque d'une absence de confiance dans la qualité de leur travail, sa légitimité reste indiscutable. En effet, il est indispensable que l'institution sache précisément comment l'école remplit les missions qui lui sont attribuées, et qui sont à la charge du contribuable. De plus, il est de son devoir de réguler les orientations prises en matière de politique éducative, dans des aspects aussi divers que les contenus d'enseignement, les plans de formation, le recrutement et la répartition des personnels, l'attribution de moyens...En d'autres termes, l'école ne pourrait pas survivre sans le contrôle institutionnel. Néanmoins, certaines questions peuvent être adressées à la manière dont ce dernier est actuellement pratiqué.

\subsection{Questions adressées aux évaluations institutionnelles}

Trois questions vont adressées. Pour cela, il est nécessaire de clarifier ce qui est entendu par contrôle, et notamment de le distinguer de la mesure des acquis.

\subsubsection{Une distinction préalable entre contrôle et mesure des acquis}


Le contrôle et la mesure des acquis ne sont pas équivalents, même si sur plusieurs aspects ils se rapprochent au point de pouvoir être parfois utilisés indifféremment. En effet, tous deux ont pour support les tâches scolaires attendues par l'institution et réalisées par l'élève : écriture de la fin d'un récit, résolution d'un problème de mathématiques, compréhension d'un texte lu... Tous deux procèdent par l'examen d'un produit fini, c'est-à-dire par le résultat de la mise en œuvre de tout un ensemble de processus et de procédures. Il est à souligner que dans les premier et second degrés, les produits sont majoritairement écrits, mais ils peuvent être oraux, gestuels, chantés, dessinés.... De plus, ni le contrôle ni la mesure des acquis ne s'intéressent à la réalisation elle-même. Ils se centrent sur un résultat dont ils donnent, à un instant T, une photographie représentée par un symbole. Qu'est-ce qui alors les distingue ?

Les travaux de la recherche en évaluation ont permis d'établir que contrôler des acquis, c'est comparer un produit réalisé par l'élève à un produit attendu. C'est établir l'adéquation d'un produit à un modèle de référence. Le contrôle déclare si le produit est conforme ou s'il ne l'est pas. Il est binaire et permet ainsi le bilan. Tandis que mesurer des acquis c'est quantifier l'adéquation du produit réalisé avec le produit attendu. Quel en est le degré de conformité ? Et donc, quelle est sa valeur ? Pour cela, une échelle d'appréciation est appliquée - qui peut être chiffrée (ce qui permet de faire des statistiques : moyennes, pourcentages...) ou présentée sous forme d'échelles ordinales (lettres, niveaux d'acquisition, appréciations assez bien, bien, très bien... - smilles, drapeaux, couleurs, gommettes...). Il est à préciser que quelle que soit l'échelle, il s'agit toujours de la même action de mesure. Cette dernière a au moins trois niveaux : elle n'est pas binaire. Elle permet la quantification de la maîtrise d'une compétence.

Quelques questions vont être adressées aux évaluations institutionnelles, qui contrôlent et mesurent des acquis?

\subsubsection{La question de la complexité des objets d'évaluation}

La première question concerne la complexité des objets d'évaluation. Le passage de l'évaluation de connaissances et de capacités, à l'évaluation des compétences, constitue une difficulté supplémentaire pour les élèves, les enseignants, les parents. Une clarification de ce qui est entendu par compétence et par évaluation de compétences semble nécessaire, comme le signale le rapport de l'IGEN (2007, p. 24, 32-33). De plus, l'évaluation par compétences peut aboutir à une dérive : la production de documents très analytiques - comme à l'époque de la pédagogie par objectifs -, dans lesquels les compétences se segmentent sur plusieurs pages où il est facile de se perdre, ce qui conduit in fine à un dessèchement du sens, 
notamment pour le cas d'élèves en difficultés ? Dans cette perspective, ne serait-il pas pertinent de «remanier» partiellement, pour les parents et pour les élèves, le livret de compétences qui est un document destiné à des professionnels ?

\subsubsection{La question de l'intensification des contrôles}

La seconde question concerne l'intensification des contrôles constatée depuis 1970 qui manifeste un souci de maîtrise de plus en plus prégnant de la part de l'institution. Cette intensification n'est-elle pas consommatrice en temps pour l'élève - notamment l'élève à BEP - comme pour l'enseignant, au détriment des temps d'enseignement-apprentissage ? Laisse-ton suffisamment de temps d'apprentissage à l'élève avant d'évaluer ses acquisitions ? De plus quel est l'effet de la succession des contrôles, sur l'estime de soi de ces élèves, dont on sait qu'elle constitue la toile de fond de l'apprentissage ?

Plus fondamentalement, l'institution utilise les évaluations nationales comme un indice de l'efficacité des enseignements. Elle postule qu'il est possible d'évaluer les enseignements à partir des résultats des élèves à ces épreuves seulement. L'institution établirait donc un lien de causalité entre la qualité des enseignements et les résultats des élèves aux contrôles institutionnels. Cette affirmation doit être questionnée, pour l'ASH notamment.

En effet, pour des élèves dont la difficulté est grave, persistante, globale, enracinée depuis longtemps, ou définitive, il est malaisé de croire que l'effet des enseignements - quelle que soit leur qualité - puisse mécaniquement se manifester, à court ou moyen terme, par une amélioration notable de leurs résultats dans des tâches d'évaluation institutionnelles standardisées. Pour autant, cela ne signifie certainement pas qu'il faille renoncer à un enseignement spécialisé, et diriger ces élèves vers un enseignement de classe ordinaire. $\mathrm{Ce}$ serait un déni de leurs caractéristiques lourd de conséquences. Cela ne signifie pas non plus que le contrôle institutionnel puisse être abandonné, car certains de ces élèves eux-mêmes souhaitent savoir, de temps à autres, où ils se situent par rapport à la norme ${ }^{9}$. Cela ne signifie pas, enfin, qu'il est impossible d'espérer des progrès de ces élèves au travers d'une amélioration de l'enseignement. Au contraire, ces progrès existent, mais ne sont pas immédiatement visibles à travers les évaluations nationales institutionnelles.

Cela signifie que les améliorations dans les apprentissages de ces élèves passent d'abord par la mise en place de compétences très finement adaptées - compétences cognitives, affectives, psycho-sociales... reliées au socle commun. Ces dernières sont progressivement repérées par l'enseignant et sont l'objet principal des projets individualisés. L'enseignement

\footnotetext{
${ }^{9}$ Norme qui, depuis fin 2006, est représentée par le socle commun de compétences.
} 
ordinaire en grand groupe, n'a, ni le temps, ni parfois l'espace, ni l'habitude, de les travailler et pourtant, elles sont la condition sine qua non des progrès. Or, ce ne sont pas celles qu'évaluent habituellement les items standardisés. Elles n'y sont donc pas observables. Ce qui peut amener à dire hâtivement que les élèves pris en charge en ASH - par exemple par les RASED - n'avancent pas suffisamment. Alors qu'en réalité, ils progressent, mais sur des aspects non mis en visibilité par les évaluations institutionnelles. En revanche, ces derniers sont perceptibles dans les évaluations menées lors des projets individualisés. Ce qui complique l'affaire, c'est qu'on ne peut les examiner qu' «à la loupe » et qu'avec un œil « spécialisé »: il faut du temps, du travail et une formation spécifique pour identifier, par exemple, certains processus cognitifs...Sinon, on risque de ne «rien y voir ». Et ce n'est que lorsque les élèves auront mis en place et relié entre elles, un petit nombre de compétences fines, c'est-à-dire à plus ou moins long terme, qu'ils pourront peut-être - mais sans certitude accéder aux compétences visées par l'institution. Il n'est donc pas rare que l'amélioration des résultats dans les contrôles institutionnels reste plus ou moins longtemps invisible, sans que la qualité et l'efficacité des enseignements et des apprentissages puisse être remise en cause.

Ce qui amène la question suivante : les résultats des évaluations institutionnelles peuventils être un instrument de pilotage suffisant du système d'Adaptation Scolarisation Handicap, du niveau local de l'école jusqu'au niveau national ? Ne gagnerait-on pas en compréhension en mettant en regard, pour ces élèves, les évaluations institutionnelles et les évaluations menées dans les projets individualisés ?

D'autres questions peuvent être adressées aux évaluations institutionnelles par le biais, cette fois-ci, des recherches en évaluation menées depuis plusieurs décennies.

\subsection{Evaluations institutionnelles et recherches en évaluation}

Nous l'avons dit, trois conceptions de l'évaluation ont été identifiées, et ceci grâce, notamment, aux travaux de Bonniol et de Vial (1997). La première, celle que nous étudions, est la mesure. Nous en retiendrons deux modèles : la métrie et la docimologie.

\begin{tabular}{|l|l|}
\hline \multicolumn{2}{|c|}{ Evaluer c'est mesurer } \\
\hline \multicolumn{2}{|c|}{ Priorité aux produits } \\
\hline \\
\hline
\end{tabular}




\begin{tabular}{|c|c|}
\hline Psychométrie Edumétrie & $\begin{array}{c}\text { Etude systématique } \\
\text { des examens }\end{array}$ \\
\hline Tests & Notation \\
\hline
\end{tabular}

De nombreuses recherches faites dans le champ de la métrie ont conduit à la construction de tests qui permettent d'évaluer, non seulement les capacités psychiques des élèves (psychométrie), mais aussi le degré d'acquisition de contenus ou compétences scolaires (édumétrie). Les tests sont très utilisés par les enseignants spécialisés et beaucoup existent en français.

Concernant la docimologie : les travaux de Noizet et Caverni (1978) et de Bonniol (1981) ont permis d'analyser expérimentalement les déterminants psychologiques de l'évaluation :

1. l'évaluation dépend du choix des critères - facettes de l'objet à évaluer que l'évaluateur choisit de privilégier parmi d'autres. Un critère est toujours relié à un monde de valeurs. D'ailleurs, étymologiquement, évaluer, c'est mettre en valeurs, c'est valoriser, c'est indiquer la valeur, c'est être en rapport avec des valeurs. Pour cela, des critères sont manipulés : c'està-dire qu'ils sont identifiés, choisis ou laissés de côté, hiérarchisés, questionnés quant à leur pertinence. Un critère « est toujours objectif, seul le choix qui en est fait est éventuellement subjectif, sans que cela signifie pour autant qu'il soit arbitraire »(Bonniol, 1981). De plus, si certains critères sont explicites, d'autres sont implicites, c'est-à-dire qu'ils opèrent efficacement sans être identifiés, et donc sans qu'il soit possible de les réguler, rendant parfois incompréhensibles certaines décisions évaluatives de l'enseignant. Les questions de l'explicitation du choix des critères et de leur hiérarchisation prennent d'autant plus d'importance que l'élève a des BEP.

2. l'évaluation dépend de l'estimation des seuils d'exigence ou de suffisance,

3. l'évaluation dépend des attentes induites par des informations extérieures sur les élèves : habillement, verbalisation, attitudes face à l'école...Ce sont des variables qui ont un rôle considérable dans la relation pédagogique, et d'autant plus avec des élèves handicapés ou en grande difficultés scolaires. Ce qui demande à l'enseignant d'avoir la capacité de rencontrer l'altérité, autrement dit, de travailler dans une éthique professionnelle.

4. l'évaluation dépend d'effets d'ordre et de contraste, dus à la succession des copies (la sévérité du correcteur varie du début à la fin de la série, en fonction des copies rencontrées...), mais aussi d' « effet de contamination de l'avis des confrères, d'effet de stéréotypie systématisant les appréciations antérieures, effet de halo des représentations sociales du candidat chez le correcteur » (Bonniol \& Vial, 1997, p. 59). 
5. l'évaluation dépend aussi de la tâche proposée et des conditions matérielles données à l'élève. Nous avons toujours à vérifier que la tâche proposée ne rende pas impossible la mise en œuvre de la compétence à évaluer. Par exemple, les élèves dyslexiques ont souvent des compétences de compréhension fine. Mais ils peuvent difficilement les mettre en œuvre à travers un texte à lire. Dans ce cas, la tentation existe de conclure rapidement qu'ils ne sont pas capables de compréhension. Alors qu'ils pourraient mobiliser cette compétence à l'oral, par exemple au travers d'un texte entendu.

6. ce n'est pas l'évaluation elle-même qui étiquette l'élève de mauvais ou de brillant. Un zéro n'a jamais dit à un élève qu' «il est nul », pas plus qu'un Non Acquis, un E, ou un Mal. C'est l'évaluateur et les personnes référentes de l'élève - camarades, amis, parents... - qui le signifient. Il revient alors à l'ingéniosité de l'enseignant, de discerner la manière et le moment où il mesurera - ou pas - les acquis de l'élève en difficultés, de manière à préserver son estime de soi, son désir d'apprendre et de persévérer. D’autres habiletés sont également requises pour accompagner certains résultats de paroles contenantes.

Toutefois, si les recherches sur la mesure permettent d'en identifier des déterminants psychologiques et de comprendre le fonctionnement du «correcteur», elles ne contribuent pas à l'amélioration des productions des élèves. Autrement dit, dès lors que la focalisation est sur le résultat d'un apprentissage, il est peu probable d'en espérer une amélioration. C'est l'une des raisons qui ont amené les chercheurs à s'intéresser à l'élève «pendant qu'il apprend ». Ce qui a permis de passer à une deuxième conception de l'évaluation.

\section{L'évaluation pour la gestion des situations d'enseignement, pour une amélioration des apprentissages.}

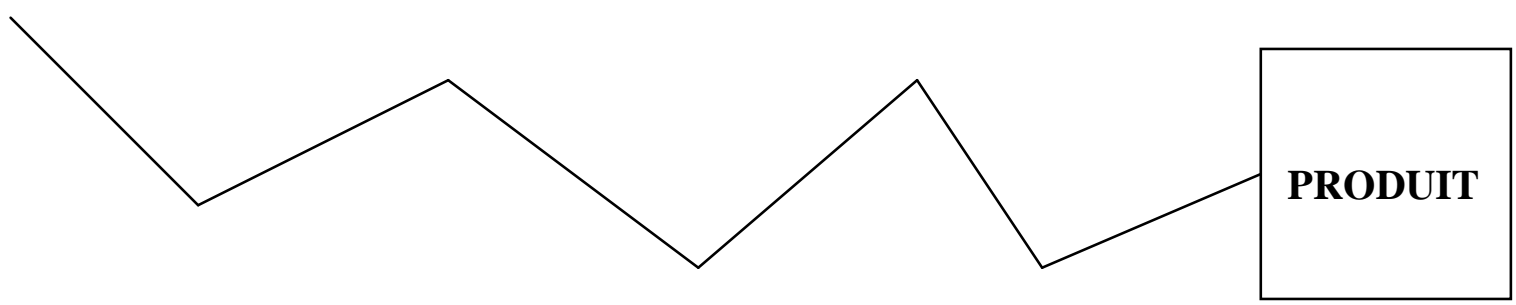

\section{PROCEDUIRES}

L'idée des chercheurs est alors de s'intéresser non plus seulement au produit, mais aux procédures qui permettent de le construire. C'est la réalisation des tâches proposées aux 
élèves, les stratégies, les techniques que ces derniers doivent mettre en place pour réussir qui sont examinées de près, et qu'on essaie de rationaliser, voire parfois de standardiser.

\begin{tabular}{|c|c|c|c|}
\hline \multicolumn{3}{|c|}{ Evaluer c'est gérer les situations d'enseignement } \\
pour améliorer les apprentissages \\
\hline \multicolumn{3}{|c|}{ Priorité aux procédures } \\
\hline $\begin{array}{c}\text { Evaluation } \\
\text { par les } \\
\text { objectifs }\end{array}$ & $\begin{array}{c}\text { Evaluation des } \\
\text { dispositifs }\end{array}$ & $\begin{array}{c}\text { Evaluation } \\
\text { formative }\end{array}$ & $\begin{array}{c}\text { Evaluation } \\
\text { formatrice }\end{array}$ \\
\hline
\end{tabular}

Les travaux de Bonniol et Vial (1997) ont identifié plusieurs modèles de cette deuxième conception de l'évaluation, dont deux figurant dans le référentiel de compétences de tout enseignant spécialisé : l'évaluation formative et l'évaluation formatrice.

\subsection{L'évaluation formative}

L'évaluation devient formative (Allal, 1979), c'est-à-dire au service de la formation. Il s'agit pour l'enseignant, d'évaluer les procédures mises en œuvre par l'élève qui réalise une tâche, afin qu'il améliore sa production. Mais que signifie évaluer des procédures ? Ce n'est pas quantifier l'adéquation du parcours de l'élève à une trajectoire attendue. Evaluer ici, c'est repérer les difficultés et les progrès de l'élève et lui donner en retour des informations sur certains aspects de son parcours pour qu'il progresse. Pour cela, l'enseignant commence par observer l'élève en train d'apprendre, sur des aspects qu'il choisit de privilégier : aspects cognitifs, affectifs, sociaux ...Il interprète ce qu'il perçoit, et prend alors des décisions sur la manière d'interagir avec lui, par exemple par le biais d'entretiens en situation. L'implication, les processus attentionnels, le rapport au savoir, le traitement de l'information, la planification de la tâche, la mémorisation, la capacité de réinvestissement des acquis...peuvent devenir pour lui des critères d'évaluation. Mais pas seulement : l'enseignant évalue aussi la manière dont l'élève ré-agit à ses interventions. Ce qui lui permet d'estimer la zone de développement proximal où les apprentissages peuvent évoluer, et par suite, de repérer ce que l'élève peut faire lorsqu'il n'est plus soutenu, et comment les acquis sont intégrés plus ou moins durablement dans une pensée cohérente. On le voit, évaluer ne signifie plus ici, pratiquer le bilan ou la mesure de ce que l'élève a produit, ni même le bilan ou la mesure de ce que l'élève est en train de faire. Il s'agit, à partir des critères d'observation choisis, de repérer les progrès 
et les difficultés des élèves, et de décider d'intervenir sur certains aspects en communiquant avec l'élève à leur propos. Par exemple, si une difficulté d'investissement dans la tâche a été repérée, l'enseignant décide d'intervenir, par une information en retour appelée « régulation »: une correction, une question, une suggestion, une information nouvelle, un autre exercice, de nouvelles conditions de travail...mais aussi en mettant en place des groupes de besoin voire de remédiation. Mais les régulations peuvent être plus fines et plus complexes. L'enseignant peut réintégrer ses objectifs de faire progresser l'élève sur les éléments repérés, en reconstituant des situations de résolution de problèmes bien choisis, ou en accompagnant les élèves dans la confrontation des productions (éventuellement la leur, mais pas nécessairement), en encore en instaurant un débat pour mettre en lumière les noeuds dont l'élève n'est pas encore suffisamment conscient. C'est ce que l'on trouve dans toutes les classes et en particulier dans l'enseignement spécialisé. Mais pour observer, analyser, interpréter l'activité des élèves, l'enseignant a toujours besoin d'un cadre de référence. Or, les cadres de référence ne sont ni identiques ni équivalents, et ils sont toujours en lien une théorie de l'apprentissage implicite ou explicite. Les conceptions de l'erreur font également partie des cadres de référence. Mais pas seulement. Pour qu'une telle analyse aboutisse, les enseignants doivent avoir recours à des modèles psychologiques et didactiques des difficultés des élèves. C'est dire les exigences de l'évaluation de l'activité des élèves à BEP, en termes d'épistémologie, de psychologie et de didactique. Ce qui pose le problème de la formation des enseignants.

\subsection{L’évaluation formatrice}

L'évaluation formatrice est «l'une des formes les plus élaborées d'évaluation formative » (Nunziati, 1990, p. 50). Au nom de «l'appropriation par les élèves des outils d'évaluation des enseignants et de la maîtrise par l'apprenant des opérations d'anticipation et de planification [...] c'est-à-dire d'une démarche de régulation de celui qui apprend » (ibid.), la priorité est de travailler avec, sur, à partir des critères et donc d'aborder les contenus de formation en cherchant, en verbalisant, en manipulant les critères des tâches pour obtenir les produits attendus.

L'autoévaluation de l'élève est définie comme le va-et-vient entre deux sortes de critères, portant sur le comment faire (critère de réalisation sur les procédures) et sur le comment je sais que c'est bien fait? (critère de réussite dans le produit). Le référentiel est donné à dévoilement (explicitation), il est peu régulable. La maîtrise des produits normés, des tâches d'examen est ici aussi une valeur indiscutable : c'est pour la réussite des produits qu'on travaille. 


\subsection{Questions adressées à l'évaluation comme gestion :}

La première question concerne la remédiation : peut-on avec des élèves suivis en ASH proposer une même remédiation à des difficultés apparemment identiques, sans les avoir analysées finement? S'agit-il comme dans le domaine médical, d'administrer un même remède pour un même symptôme ? Et quelles sont les conséquences pour ces élèves, d'une standardisation de la remédiation?

La seconde question concerne un constat établi par le rapport IGEN de 2007. Concernant l'enseignement ordinaire, il est souligné que «L'évaluation formative elle-même n'est guère plus performante. Les outils habituellement utilisés par les enseignants ne permettent que rarement de repérer les difficultés naissantes et d'intervenir sans retard pour éviter qu'elles ne s'aggravent. » (2007, p. 32). S'il est établi que les enseignants de classe ordinaire ne repèrent que rarement difficultés naissantes et ne la préviennent pas assez rapidement, pourquoi continuent-ils à gérer, sans aucune aide nouvelle, les difficultés ponctuelle et moyenne? Les deux heures hebdomadaires de soutien scolaire y suffiront-elles à elles seules ? De plus, comment pourront-ils aider, accompagner les élèves en grandes difficultés, dans des écoles où les enseignants spécialisés semblent devoir être de moins en moins nombreux ?

\section{L'évaluation comme questionnement du sens de ce qui est dit et de ce qui est fait}

Le troisième modèle s'intéresse à l'évaluation conçue comme volonté de rendre intelligible ce qu'on fait. L'intelligibilité est ce mouvement de production de sens sur ce qui se dit et sur ce qui se fait en situation, mouvement qui suscite la réflexion et la critique - un travail des valeurs.

Pour l'enseignant, un travail d'évaluation comme questionnement du sens est enclenché lorsqu'un événement vient le «dé-router» de la direction qu'il a prise pour atteindre ce qui est demandé, ce qui est attendu, ce qui a été commencé. Et l'une des particularités des situations d'enseignement est qu'elles sont traversées par un grand nombre d'événements. Ce qui avait été initialement prévu n'est plus tout à fait adéquat. Il lui faut faire partiellement autrement, voire autre chose que ce qu'il sait déjà faire, que ce qui est schématiquement prédéfini. De plus, la plupart du temps, il n'existe aucun ensemble ordonné de prescriptions, de normes - aucun algorithme préétabli à appliquer mécaniquement - qui lui permettraient d'agir à ce moment-là. Il doit alors réguler pour partie son dispositif. Il mobilise son 
intelligence des situations et puise dans les ressources de son expérience, de son histoire, de sa volonté, pour interpréter ce qui se joue, pour rappeler les valeurs professionnelles et se travailler avec elles. Il est amené à problématiser ce qu'il fait, pour se donner de nouvelles normes d'agir, c'est-à-dire pour renormaliser (Schwartz, 2004) de manière à réguler son action, et trouver d'autres manières de faire. Pour cela, il auto-évalue très rapidement son travail, sa pratique, la pertinence de ses interventions. Dans cette situation, se posent avec une acuité particulière les questions du sens de ses pratiques, de ses relations avec les élèves. Il n'y a pas de moment prévu pour ce type d'évaluation qu'on appelle « évaluation située », qui reste le plus souvent dans le for intérieur de l'enseignant, clandestine, peu verbalisée, et par conséquent peu connue et reconnue. Et pourtant c'est certainement la plus fréquente. Elle est indispensable à l'enseignement.

Pour les élèves aussi, en fonction de leur âge, et notamment pour les élèves suivis en ASH, se pose à certains moments cruciaux la question du sens de ce qu'ils disent et font, ce qui les met en rapport avec des valeurs sociales. Ce sont des moments de discussion fugitifs réitérés, d'apparence anodine voire futile, des intrusions plus ou moins fréquentes dans la séquence didactique. C'est pour cette raison que le travail d'intelligibilité est le plus souvent imprévu, inattendu, de l'ordre de la surprise, et qu'il a des modes de manifestation particuliers.

\begin{tabular}{|c|c|}
\hline \multicolumn{2}{|c|}{$\begin{array}{l}\text { Evaluer c'est problématiser le sens de ce qui est dit, } \\
\text { de ce qui est fait }\end{array}$} \\
\hline \multicolumn{2}{|c|}{ Priorité aux auteurs, aux personnes } \\
\hline La pensée complexe & L'herméneutique \\
\hline
\end{tabular}

\section{Conclusion}

Ce rapide exposé des trois conceptions de l'évaluation, et des modèles qui leur sont attachés, montrent que c'est un objet hétérogène, une pratique polymorphe qui est loin de se réduire à la seule mesure des acquis. L'enseignant évalue certes pour quantifier la maîtrise de compétences, mais aussi pour améliorer les enseignements-apprentissages, et pour réfléchir au sens de ce qui est dit et fait. L'évaluation habite ainsi la totalité de la situation éducative.

Dans tous les cas, il s'agit pour l'enseignant d'établir des hiérarchisations de critères qu'il a la responsabilité de choisir parmi d'autres. Ces choix et ces hiérarchisations sont des arbitrages, des prises de décision qui résultent de débats de normes (Schwartz, 2004) et de délibérations de valeurs qui ne sont jamais écrits à l'avance et qui l'impliquent chaque fois 
profondément. En ASH, ces débats prennent un relief particulier puisque les critères doivent être finement adaptés aux caractéristiques cognitives, psychologiques, affectives et sociales de chacun des élèves, ce qui peut les rendre imperceptibles à un œil «non spécialisé ». Dans ces arbitrages, «il y a toujours une sorte de destin à vivre » (Schwartz, 2003, p.187) sachant qu'il y le risque d'échouer et par conséquent, la nécessité d'assumer les conséquences de ses choix. La vigilance constante aux choix des critères et à leur hiérarchisation, le souci de l'évaluateur de remonter le fil des débats de normes épistémologiques et didactiques, de repérer les valeurs philosophiques, éthiques et politiques qui y ont présidé, contribue à donner à l'évaluation le caractère humaniste qui joue un rôle considérable auprès des élèves à BEP.

Bibliographie

Allal, L. (1979) Stratégies d'évaluation formative : conception psycho-pédagogique et modalités d'application. Allal, L., Cardinet, J., Perrenoud, P., L'évaluation formative dans un enseignement différencié, Berne : Peter Lang, pp.129-156.

Bonniol, J-J. (1981) Déterminants et mécanismes des comportements d'évaluation des épreuves scolaires, thèse de doctorat ès Lettres et Sciences Humaines, Bordeaux, 1981.

Bonniol, J-J. \& Vial, M. (1997) Les modèles de l'évaluation, textes fondateurs et commentaires. Bruxelles : De Boeck Université.

Cardinet, J. (1979). L'élargissement de l'évaluation. Education et recherche, 1(1), pp. 15-34.

Hadji, C. (1990 2 ed) L'évaluation, règles du jeu, Paris, ESF, 1989.

Lecointe, M. (1997) Les enjeux de l'évaluation. Paris : L'Harmattan.

Ministère de l'Education Nationale (2007): Mise en œuvre du cahier des charges de la formation des maitres, Circulaire. $n^{\circ}$ 2007-045 du 23-2-2007, NOR MEN BO 0700441C, Référence : Arrêté du 19-12-2006.

Noizet, G \& Caverni, J-P. (1978) Psychologie de l'évaluation scolaire, Paris, PUF.

Nunziati, G. (1990) Pour construire un dispositif d'évaluation formatrice. Les Cahiers pédagogiques, $\mathrm{n}^{\circ} 280$. pp.48-64.

Schwartz, Y. (2004) L'expérience est-elle formatrice ? Education Permanente ${ }^{\circ}$ 158, pp. 11 24.

Schwartz, Y. \& Durrive, L. (2003) Travail \& Ergologie, Entretiens sur l'activité humaine. Toulouse : Octarès 
Vial, M. (2001) Se former pour évaluer, se donner une problématique et élaborer des concepts. Bruxelles : De Boeck Université. 